\title{
The Legacy of Daniel Quillen
}

\section{Foreword}

This issue of the Journal of K-Theory is dedicated to the legacy of Daniel Quillen (1940-2011), who created many of the subjects covered by our journal. The issue comprises nine essays on some of Quillen's major contributions to algebra, geometry and topology. Each essay is based on one or more of Quillen's major articles; we have ordered the essays so that they roughly follow the chronology of Quillen's publications. The topics treated include Quillen model categories, abstract homotopy theory, rational homotopy theory, formal group laws and complex cobordism, the spectrum of group cohomology, the Adams conjecture, higher algebraic K-theory, Serre's problem, algebraic K-theory and cyclic homology, and the foundations of cyclic (co)homology.

Certainly Quillen was both a prodigious problem solver and theory builder, rolled into one person. This happens very seldom. His solution of the Adams conjecture and his development of higher algebraic K-theory provide an example of these two styles; moreover the role of the former problem in creating the latter theory shows how intimately problem solving and theory building were intermingled and integrated in his work.

More than anything else, he was magnificently creative. His ability to transfer concepts, constructions and methodology from one area of mathematics to others allowed him to connect existing fields in totally unexpected ways, and also to create new landscapes. The most notable of his creations was higher algebraic K-theory, where the topics and disciplines of algebra, geometry, and topology converge in a harmonious whole which provides new tools for investigating old problems and also creates new research areas.

The editors of the Journal of K-Theory humbly take this opportunity to thank Dan for his unwavering support and encouragement in bringing about our discipline as well as our journal, and for his service as editor on K-Theory, the forerunner of our journal, during its entire lifetime.

Anthony BaK, Jonathan Rosenberg, And Charles Weibel 


\author{
Institute for Advanced Study \\ Academic Year 1969 - 1970 \\ (year of the Adams conjecture and the plus construction)
}

from left to right

George Lusztig, Daniel Quillen, Graeme Segal, Michael Atiyah 


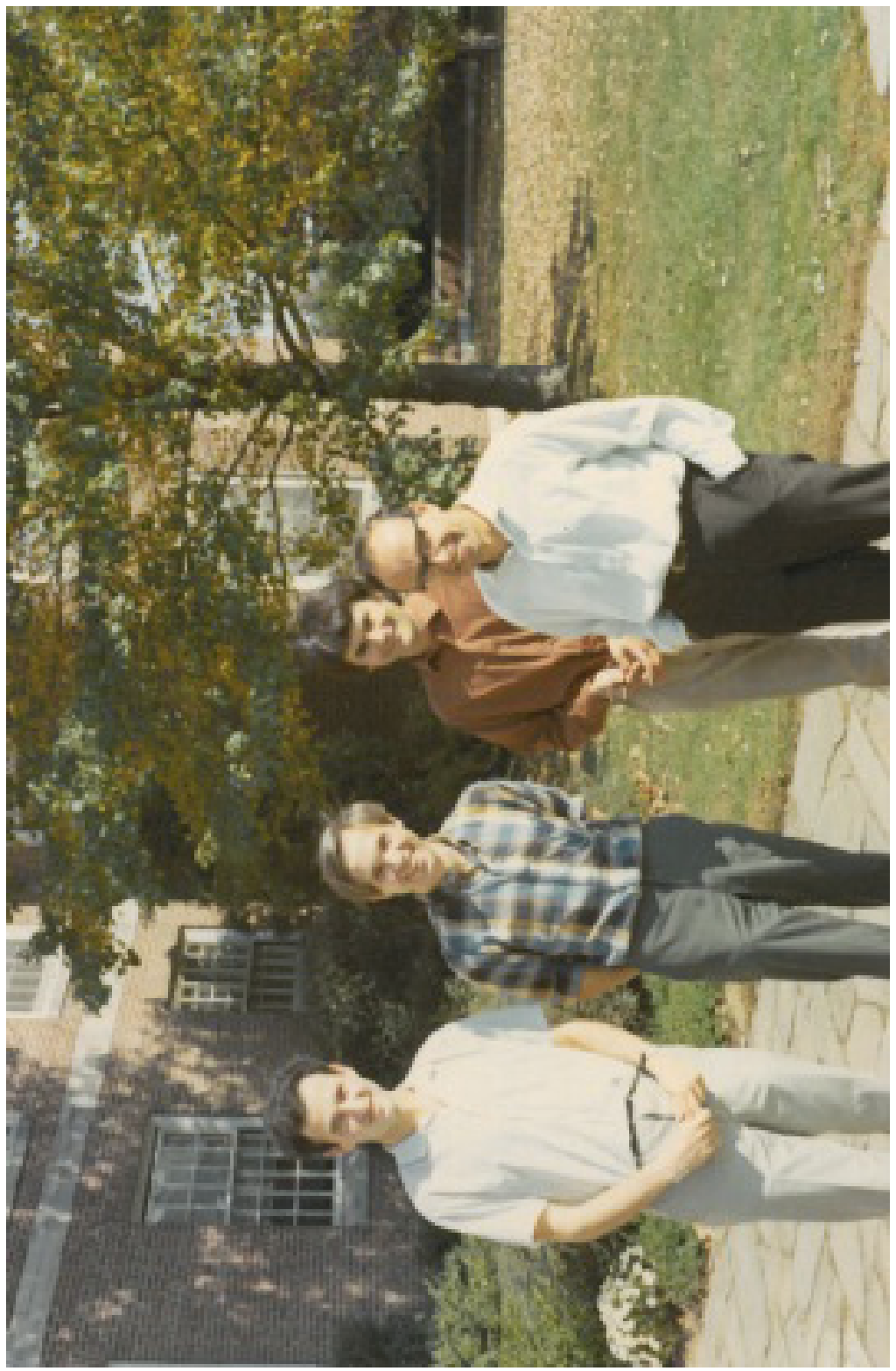

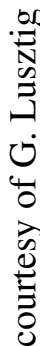

